\title{
Celiac disease case finding strategy in Romanian symptomatic children
}

\author{
Gabriela Lesanu ${ }^{1,2}$, Raluca Maria Vlad ${ }^{1,2}$, Cristina Becheanu ${ }^{1,2}$, Iulia Florentina Tincu1, \\ Olivia Dragnescu ${ }^{3}$, Alexandra Coroleuca ${ }^{1}$, Radu George Nicolaescu ${ }^{4}$, Daniela Pacurar ${ }^{1,2}$ \\ 1 "Grigore Alexandrescu" Emergency Children's Hospital, Bucharest, Romania \\ 2 "Carol Davila" University of Medicine and Pharmacy, Bucharest, Romania \\ 3 “Alexandru Trestioreanu” Oncological Institute, Bucharest, Romania \\ ${ }^{4}$ Fundeni Clinical Institute, Bucharest, Romania
}

\begin{abstract}
Objectives. Pediatricians face various challenges in different stages of celiac disease (CD) diagnosis. Whom to test is intensely debated because of CD's heterogenous clinical spectrum. The main purpose of the study was to identify symptoms or symptom associations that should initiate an active strategy of CD early diagnosis in Romanian children.

Material and method. We conducted a prospective study in "Grigore Alexandrescu" Emergency Children's Hospital. From March 2013 until February 2014, 249 children with symptoms/signs at risk of CD were included. Results. CD was diagnosed in 11 (1/21 patients evaluated). One in 12.6; 16; $18 ; 18.5$ and 18.5 children respectively with chronic diarrhea, low stature, growth failure, recurrent abdominal pain and constipation had CD. Certain symptom associations increased the risk: classical symptom associations (chronic diarrhea and weight loss), as well as other associations: recurrent abdominal pain and weight loss, constipation and weight loss, constipation and refractory iron deficiency anemia.

Conclusion. Active screening among patients with symptoms and especially symptom associations at risk of $C D$ would improve diagnosis rates in pediatric $C D$.
\end{abstract}

Keywords: Celiac disease, children, active screening

\section{INTRODUCTION}

Several recent studies show that the incidence of celiac disease $(\mathrm{CD})$ is still rising (1-3). Although the medical community awareness towards $\mathrm{CD}$ is high, a large number of cases are not diagnosed. We are facing several challenges in different stages of CD diagnosis.

The first issue remains the identification of cases that require serological screening. Whom to test is yet intensely debated because CD has a heterogenous clinical spectrum and even more, the clinical presentation changed significantly in the last decades: the prevalence of the classical form decreased, while cases with extraintestinal symptoms or less suggestive gastrointestinal symptoms are increasingly more frequent (4-8).

Our statistics show a decrease in the number of cases diagnosed in the hospital. An explanation might be related to the implication of the primary care and family doctors in the identification of symptomatic cases through serological screening, followed by complete diagnosis according to the ESPGHAN criteria (9) without histological examination. In these instances, some cases might escape serological screening because of the atypical clinical presentation.

An effective case finding strategy is necessary in order to decrease the morbidity and mortality generated by untreated CD (10). Our goal was to identify symptoms or most frequent symptom associations that should raise the suspicion of $\mathrm{CD}$ and initiate an active strategy of early diagnosis.

\section{MATERIAL AND METHOD}

We conducted a prospective study in "Grigore Alexandrescu" Emergency Children's Hospital, 
Bucharest, Romania, that included patients hospitalised in a 12 months interval, from March 2013 until February 2014. All cases admitted in the Pediatric Departments (Gastroenterology, Pneumology, Toxicology and General Pediatrics) were evaluated in order to identify patients with signs and symptoms suggestive of CD.

The inclusion criteria were gastrointestinal and/ or non-gastrointestinal symptoms comprised in the 2012 ESPGHAN diagnostic guideline (9). A group of trained pediatric specialists and residents recorded patients' data in an inclusion form (demographics, alimentation history, signs and symptoms, nutritional status).

For each patient, the IgA antitransglutaminase antibodies (ATG) and the seric IgA were determined. For patients with IgA deficiency, the level of IgG-ATG was used instead.

Diagnosis of CD was established according to revised ESPGHAN criteria, 2012 (9). Intestinal biopsy was indicated and performed to all patients with positive ATG, but with levels less than 10 fold the normal value. The Marsh-Oberhuberand classification was used.

For patients with ATG more than 10 fold the normal value, anti-endomysial antibodies (EMA) and HLA DQ2/DQ8 were performed. In these cases the duodenal mucosal biopsy was optional after a previous discussion with the parents.

A single/multivariant statistical analysis was performed to identify risk symptoms and symptom associations.

The study was approved by the Ethics Committee of „Grigore Alexandrescu” Emergency Children's Hospital. An informed consent was obtained from the parents of each eligible patient.

\section{RESULTS}

Between $1^{\text {st }}$ of March 2013 and $28^{\text {th }}$ of February 2014, 9740 patients were admitted in the four Departments of Pediatrics of "Grigore Alexandrescu" Emergency Children's Hospital (Gastroenterology, Pneumology, Toxicology and General Pediatrics). Out of these, 249 children with symptoms/signs at risk of CD according to ESPGHAN guideline (9) were selected and included in the study (124 girls, 125 boys, mean age: 5 years 4 months). Table 1 illustrates the clinical presentation of the patients included.

Eight children had IgA deficiency and were tested for IgG-ATG. IgA-ATG were positive in 11 patients and 3 IgA deficient children had high IgGATG. Out of 14 patients with positive ATG (IgA/
$\mathrm{IgG}), 2$ were lost from follow-up (they did not come back to complete the investigation protocol).

TABLE 1. Symptoms/signs at risk of $C D$ the patients included in the study

\begin{tabular}{|l|c|c|}
\hline Symptoms/signs & $\begin{array}{c}\text { No. } \\
\text { cases }\end{array}$ & Percentage \\
\hline Chronic diarrhea & 63 & 25.3 \\
\hline Failure to thrive & 147 & 59 \\
\hline Short stature & 49 & 19.6 \\
\hline Weight loss & 18 & 7.2 \\
\hline Abdominal distention/bloating & 23 & 9.2 \\
\hline Constipation & 37 & 14.8 \\
\hline Recurrent abdominal pain & 51 & 20.4 \\
\hline Recurrent vomiting & 12 & 4.8 \\
\hline Anorexia & 45 & 18 \\
\hline Recurrent stomatitis & 5 & 2 \\
\hline Chronic fatigue & 10 & 4 \\
\hline Behaviour changes & 2 & 0.8 \\
\hline Delayed onset of puberty & 2 & 0.8 \\
\hline Increased level of liver enzymes & 14 & 5.6 \\
\hline Refractory iron-deficiency anemia & 8 & 3.2 \\
\hline
\end{tabular}

Four patients had ATG less than 10 fold the normal value and intestinal biopsy was performed showing Marsh 3 changes, which confirmed the diagnosis of $\mathrm{CD}$. For these 4 patients, the genetic testing was also performed and all were HLA DQ2 positive.

Eight patients had ATG more than 10 fold the normal value. All 8 underwent genetic testing: one proved HLA DQ2/DQ8 negative and the diagnosis of CD was excluded (false positive ATG); the other 7 tested positive for HLA DQ 2 (4 patients) or HLA DQ 8 (2 patients) or HLA DQ 2 and DQ 8 (1 patient). EMA was performed for all these patients with high ATG, testing positive and therefore, corroborated with the positive HLA testing, the diagnosis of CD was confirmed. For three patients with high ATG, intestinal biopsy was also performed and Marsh 3 lesions were detected.

CD was diagnosed in 11 children ( 9 girls, 2 boys), representing $1 / 21$ patients presenting with at risk symptoms. Mean age at diagnosis was 4 years and 1 month \pm 1 year and 7 months.

The age gluten was introduced for the celiac patients was in average 7.3 months, ranging from 4 to 12 months.

The most frequent presenting symptoms for celiac patients were: failure to thrive, chronic diarrhea, constipation and recurrent abdominal pain (Table 2).

One in $12.6 ; 16 ; 18$ children with chronic diarrhea, low stature, growth failure, but also 1 in 18.5 with recurrent abdominal pain and constipation respectively had CD. 
TABLE 2. Presenting symptoms/signs in children diagnosed with $C D$

\begin{tabular}{|l|c|c|}
\hline Symptoms/signs & $\begin{array}{c}\text { No. } \\
\text { cases }\end{array}$ & Percentage \\
\hline Failure to thrive & 8 & 72 \\
\hline Chronic diarrhea & 5 & 45.5 \\
\hline Constipation & 4 & 36.4 \\
\hline Recurrent abdominal pain & 4 & 36.4 \\
\hline Short stature & 3 & 27.3 \\
\hline Weight loss & 3 & 27.3 \\
\hline Recurrent vomiting & 3 & 27.3 \\
\hline Abdominal distention/bloating & 2 & 18.2 \\
\hline Anorexia & 1 & 9.1 \\
\hline Behaviour changes & 1 & 9.1 \\
\hline Increased level of liver enzymes & 1 & 9.1 \\
\hline Refractory iron-deficiency anemia & 1 & 9.1 \\
\hline
\end{tabular}

A number of symptom associations were demonstrated to have put the patient at high risk of CD (Table 3).

TABLE 3. Associations of symptoms and the risk of $C D$

\begin{tabular}{|l|c|c|c|}
\hline Associations of symptoms & OR & 95\% Cl & P \\
\hline $\begin{array}{l}\text { Recurrent abdominal pain and } \\
\text { weight loss }\end{array}$ & 29.3 & $5.1-168.8$ & 0.001 \\
\hline Chronic diarrhea and weight loss & 27.4 & $14.4-52.1$ & 0.003 \\
\hline $\begin{array}{l}\text { Chronic diarrhea and failure to } \\
\text { thrive }\end{array}$ & 8.6 & $2.4-30.6$ & 0.003 \\
\hline Constipation and weight loss & 26.2 & $3.3-207.7$ & 0.01 \\
\hline Constipation and refractory anemia & 24.8 & $13.5-45.5$ & 0.04 \\
\hline
\end{tabular}

\section{DISCUSSIONS}

Out of 249 patients (mean age: 5 years and 4 months, ranging from 8 months to 17 years) presenting with symptoms/signs compatible with the diagnosis of celiac disease, 14 patients were identified to have positive ATG (mean age: 4 years, ranging from 1 year and 11 months to 8 years). Although we evaluated children of all ages, the majority (71.4\%) of children with positive celiac serology were younger than 6 years.

$\mathrm{CD}$ was diagnosed in 1 out of 21 children presenting with at risk symptoms. The children diagnosed with CD (11 patients) had the mean age of 4 years and 1 month, ranging from 1 year and 11 months to 6 years and 11 months. The majority (9 cases) were female, although similar percentages of boys and girls underwent celiac serology testing.

The children of this cohort were born between 1996 and 2012. For the majority of them, the gluten was introduced later, according to ESPGHAN recommendations at that time; these were only modified in 2008 (11). Gluten is recommended to be introduced progressively between 4 and 7 months. In our study, in celiac patients the gluten was intro- duced between 6 and 12 months, with one exception (4 months). The delayed introduction of gluten is associated with delayed onset of the disease; this is an old observation, but is sustained by recent studies as well (12).

Less than half of celiac patients presented with the classical form of the disease (chronic diarrhea and failure to thrive). None of them had celiac crisis, nor severe malnutrition, clinical presentations we have been used to until a few years back (13). The other patients diagnosed with celiac disease presented with recurrent abdominal pain, constipation, failure to thrive and one patient with increased liver enzymes. Milder, mono or oligosymptomatic forms became more frequent in our experience too, similar to reports from other European countries $(7,8,14)$.

Out of 63 children (ranging from 7 months to 15 years) presenting with chronic diarrhea, five (7.9\%) were celiac (ranging from 1 year and 11 months to 4 years and 8 months). So, chronic diarrhea is still a characteristic presenting manifestation of celiac disease in young children, other recently publicated studies confirming this finding $(15,16)$. Severe chronic diarrhea with steatorrhea is now rarely reported, often patients presenting with intermittent, recurrent diarrhea that should raise the pediatrician's clinical suspicion and lead to screening with celiac serology. The risk of celiac disease is higher when chronic diarrhea is associated with weight loss.

One or two decades ago, recurrent abdominal pain was rarely associated with celiac disease and for this presentation, serology screening was not considered to be justified (17). Later, this became one of the frequently seen clinical presentation in Western European countries, North America (6,18$20)$ and other geographical areas $(21,22)$ as a single clinical manifestation or in association with other symptoms. In the study of Khatib that looked at a group of celiac children recently diagnosed (20032013) in United States, abdominal pain was reported in higher percentages than diarrhea, being the most frequent digestive symptom (23). In our study, $7.8 \%$ of patients with recurrent abdominal pain had celiac disease. The risk of CD is even higher when recurrent abdominal pain is associated with weight loss.

Constipation has been reported for many years in celiac patients, but the frequency is different in various studies. The multicentric study TEDDY showed that in symptomatic celiac children, the constipation was as frequent as diarrhea (16). The indication of screening with celiac serology for children with constipation is still controversial, not 
being recommended by some (24), but considered necessary by others (25). Out of 37 children with constipation, four $(10.8 \%)$ had $\mathrm{CD}$; our study suggests that the risk of CD increases when constipation is associated with other symptoms/signs (recurrent abdominal pain, refractory iron-deficiency anemia or weight loss).

Out of 147 patients with failure to thrive, eight were diagnosed with celiac disease; 6 of these associated other symptoms, but for 2 this was the only manifestation.

Considering that $\mathrm{CD}$ has a high prevalence, but the majority of patients remain undiagnosed because of the difficult clinical detection, lately the utility of screening is debated (26). A recent paper analyzed CD from the perspective of World Health Organization (WHO) criteria for mass screening and the authors concluded that current evidence is not sufficient to support mass screening for $\mathrm{CD}$, but active case-finding may be appropriate (27). The case finding strategy we used in the present study led to an increase in the incidence of $\mathrm{CD}$ diagnosis in our Pediatric Departments from 0.28 patients diagnosed with CD in 1000 admissions in the preceding year to 1.13 in 1000 admissions in the year of the study.

\section{REFERENCES}

1. West J., Fleming K.M., Tata L.J. et al. Incidence and prevalence of celiac disease and dermatitis herpetiformis in the UK over two decades: population-based study. Am J Gastroenterol. 2014 May; 109(5):757-68;

2. Altobelli E., Paduano R., Petrocelli R., Di Orio F. Burden of celiac disease in Europe: a review of its childhood and adulthood prevalence and incidence as of September 2014. Ann Ig. 2014 Nov-Dec; 26(6):485-98;

3. Tapsas D., Hollén E., Stenhammar L., Fälth-Magnusson K. Unusually High Incidence of Paediatric Coeliac Disease in Sweden during the Period 1973 - 2013. PLoS One. 2015 Dec 11; 10(12):e0144346;

4. Whyte L.A., Jenkins H.R. The epidemiology of coeliac disease in South Wales: a 28-year perspective. Arch Dis Child. 2013 Jun; 98(6):405-7;

5. Green P.H., Krishnareddy S., Lebwohl B. Clinical manifestations of celiac disease. Dig Dis. 2015; 33(2):137-40;

6. Tolone C., Pellino V., Piccirillo M., et al. Recurrent abdominal pain in children: underlying pathologies in absence of alarm symptoms and signs in a tertiary center. Minerva Pediatr. 2016 Jan 8 [Epub ahead of print];

7. Gokce S., Arslantas E. Changing face and clinical features of celiac disease in children. Pediatr Int. 2015 Feb; 57(1):107-12;

8. Tapsas D., Hollén E., Stenhammar L., Fälth-Magnusson K. The clinical presentation of coeliac disease in 1030 Swedish children: Changing features over the past four decades. Dig Liver Dis. 2016 Jan; 48(1):16-22;

9. Husby S., Koletzko S., Korponay-Szabo I.R. et al. for the ESPGHAN Working Group on Coeliac Disease Diagnosis, on behalf of the ESPGHAN Gastroenterology Committee: European Society for Pediatric Gastroenterology, Hepatology and Nutrition Guidelines for

\section{CONCLUSIONS}

A $4.4 \%$ prevalence of $\mathrm{CD}$ was observed in symptomatic patients.

Chronic diarrhea is still a frequent clinical presentation for CD patents, especially in young children, but almost equally as frequent are recurrent abdominal pain or constipation, therefore celiac serology screening should be also considered for children with these symptoms in our country as well.

Certain symptom associations increase the risk of CD: classical symptom associations (chronic diarrhea and weight loss) as well as other associations (recurrent abdominal pain and weight loss, constipation and weight loss, constipation and refractory iron deficiency anemia) were demonstrated most often in patients with CD.

Active screening among patients with symptoms and especially symptom associations at risk of $\mathrm{CD}$ would improve the diagnosis rates in pediatric $\mathrm{CD}$.

Conflict of interest: none declared Financial support: none declared

the diagnosis of coeliac disease - J Pediatr Gastroenterol Nutr. 2012; 54(1): 136-60;

10. Catassi C., Gatti S., Lionetti E. World perspective and celiac disease epidemiology. Dig Dis. 2015; 33(2):141-6;

11. Agostoni C., Decsi T., Fewtrell M. et al. ESPGHAN Committee on Nutrition: Complementary feeding: a commentary by the ESPGHAN Committee on Nutrition, J Pediatr Gastroenterol Nutr. 2008 Jan; 46(1):99-110;

12. Lionetti E., Castellaneta S., Francavilla R., et al. SIGENP (Italian Society of Pediatric Gastroenterology, Hepatology, and Nutrition) Working Group on Weaning and CD Risk.: Introduction of gluten, HLA status, and the risk of celiac disease in children, N Engl J Med. 2014 Oct 2; 371(14):1295-303;

13. Lesanu G., Costea F., Hurduc C. et al. Changing pattern of presentation of celiac disease Arch. Dis Child 2008; 93 (Suppl II) A152;

14. Lurz E., Scheidegger U., Spalinger J. et al. Clinical presentation of celiac disease and the diagnostic accuracy of serologic markers in children. Eur J Pediatr. 2009 Jul; 168(7):839-45;

15. Kho A., Whitehead M., Day A.S. Coeliac disease in children in Christ Church, New Zealand: Presentation and patterns from 2000-2010. World J Clin Pediatr. 2015 Nov 8;4(4):148-54;

16. Agardh D., Lee H.S., Kurppa K. et al. TEDDY Study Group.: Clinical features of celiac disease: a prospective birth cohort. Pediatrics. 2015 Apr; 135(4):627-34;

17. Fitzpatrick K.P., Sherman P.M., Ipp M. et al. Screening for celiac disease in children with recurrent abdominal pain. $J$ Pediatr Gastroenterol Nutr. 2001 Sep; 33(3):250-2;

18. McGowan K.E., Castiglione D.A., Butzner J.D. The changing face of childhood celiac disease in north america: impact of serological testing. Pediatrics. 2009 Dec;124(6):1572-8. doi: 10.1542/peds.2008-2373; 
19. Green P.H., Krishnareddy S., Lebwohl B. Clinical manifestations of celiac disease. Dig Dis. 2015; 33(2):137-40;

20. White L.E., Bannerman E., McGrogan P. et al. Childhood coeliac disease diagnoses in Scotland 2009-2010: the SPSU project. Arch Dis Child. 2013 Jan; 98(1):52-6;

21. Iwańczak B., Matusiewicz K., Iwańczak F. Clinical picture of classical, atypical and silent celiac disease in children and adolescents. Adv Clin Exp Med. 2013 Sep-Oct; 22(5):667-73;

22. Sarkhy A.A., El Mouzan M.I., Saeed E. et al. Clinical Characteristics of Celiac Disease and Dietary Adherence to Gluten-Free Diet among Saudi Children. Pediatr Gastroenterol Hepatol Nutr. 2015 Mar; 18(1):23-9;

23. Khatib M., Baker R.D., Ly E.K. et al. Presenting Pattern of Pediatric Celiac Disease. J Pediatr Gastroenterol Nutr. 2016 Jan; 62(1):60-3;
24. Dehghani S.M., Ehsaei Z., Honar N., Javaherizadeh H. Frequency of Celiac Disease In Children With Chronic Functional Constipation in Shiraz-Iran. Middle East J Dig Dis. 2015 Jul; 7:166-9;

25. Pelleboer R.A., Janssen R.L., Deckers-Kocken J.M., et al. Celiac disease is overrepresented in patients with constipation. $J$ Pediatr (Rio J). 2012 Mar-Apr; 88(2):173-6;

26. Catassi C., Fasano A. Coeliac disease. The debate on coeliac disease screening - are we there yet? Nat Rev Gastroenterol Hepatol. 2014 Aug; 11(8):457-8;

27. Ludvigsson J.F., Card T.R., Kaukinen K. et al. Screening for celiac disease in the general population and in high-risk groups. United European Gastroenterol J. 2015 Apr; 3(2):106-20. 